\title{
Endotracheal Intubation and Head Posture in Infants
}

\author{
Y. K. BOSMAN. P. A. FOSTER
}

\begin{abstract}
SUMMARY
The movement of an endotracheal tube relative to the carina has been studied with the head in different positions. The dangers of malposition and displacement are stressed. Some precautions necessary to avoid these hazards are given.
\end{abstract}

S. Afr. med. J., 52, 71 (1977).

In adults, malposition of an endotracheal tube is a hazard with which the anaesthetist or staff in intensive care units must learn to live. Many writers have emphasized this: 'Improper placement of endotracheal catheters is not at all uncommon', or 'Repeated instances of endobronchial intubation have been discovered in which auscultation of the chest revealed only equivocal evidence', ${ }^{1}$ or 'Inadvertent intubation of the right main bronchus is commoner than might be supposed'.

In infants endotracheal intubation poses an even greater challenge. Kuhns and Poznanski ${ }^{3}$ found that the tube was beyond the carina in 18 out of 36 infants who had been intubated. Atelectasis occurred in 11 of these, but it developed only if the endotracheal tube was more than $0,5 \mathrm{~cm}$ into the bronchus. In all these patients emergency intubations had been done by interns and residents for life-threatening conditions. The same authors also showed movement of the endotracheal tube tip towards the carina with flexion of the head, and movement away from the carina on rotation. No X-ray film had been taken with the head in full extension. In a study of intubations done by paediatric house officers, the tube was found to be in the right main bronchus in 9 out of 14 patients. ${ }^{4}$

This investigation was undertaken to determine the importance of changes of head position as a contributory cause of endobronchial intubation or accidental extubation.

\section{SUBJECTS AND METHODS}

This study was done on 2 infants who had died shortly before the experiment. The one infant weighed $3,4 \mathrm{~kg}$ and was first intubated nasally with a No. 3,0 rubber nasotracheal tube. The tube had a pin marking its distal end, and was firmly fixed at the nostril. Radiographs were taken with the head in mid-position, in full flexion, in extension and in rotation. The carina was marked by a radiologist. The distance, in millimetres, between the carina and the tube tip is indicated on each X-ray film (Figs 1-4).

\footnotetext{
Department of Anaesthetics, University of Stellenbosch and Tygerberg Hospital, Parowvallei, CP

Y. K. BOSMAN, M.B. B.CH., F.F.A. (S.A.)

P. A. FOSTER, M.B. CH.B., F.F.A., D.A.

Date received: 13 December 1976.
}

Maximum movement of the tip of the tube was seen between full flexion and full extension. When the X-ray film with the head in full extension was taken, the chest and pelvis were raised $10 \mathrm{~cm}$ nearer to the $\mathrm{X}$-ray tube in order to include the skull on the radiograph. This introduced a magnification of the distance from the tube tip to the carina of $\pm 7 \%$, for which no correction was made in the measurement.

The dead infant was then intubated orally with the same tube, which was fixed to the lower lip. Radiographs were again taken with the head in the same postures. With the head in extension and rotation, the mouth was in the half-open position, which accounts for the lesser up and down movement in comparison with the situation pertaining in nasal intubation.

In a second subject, also an infant that had recently died and that weighed $7,1 \mathrm{~kg}$, the same series of radiographs were taken.

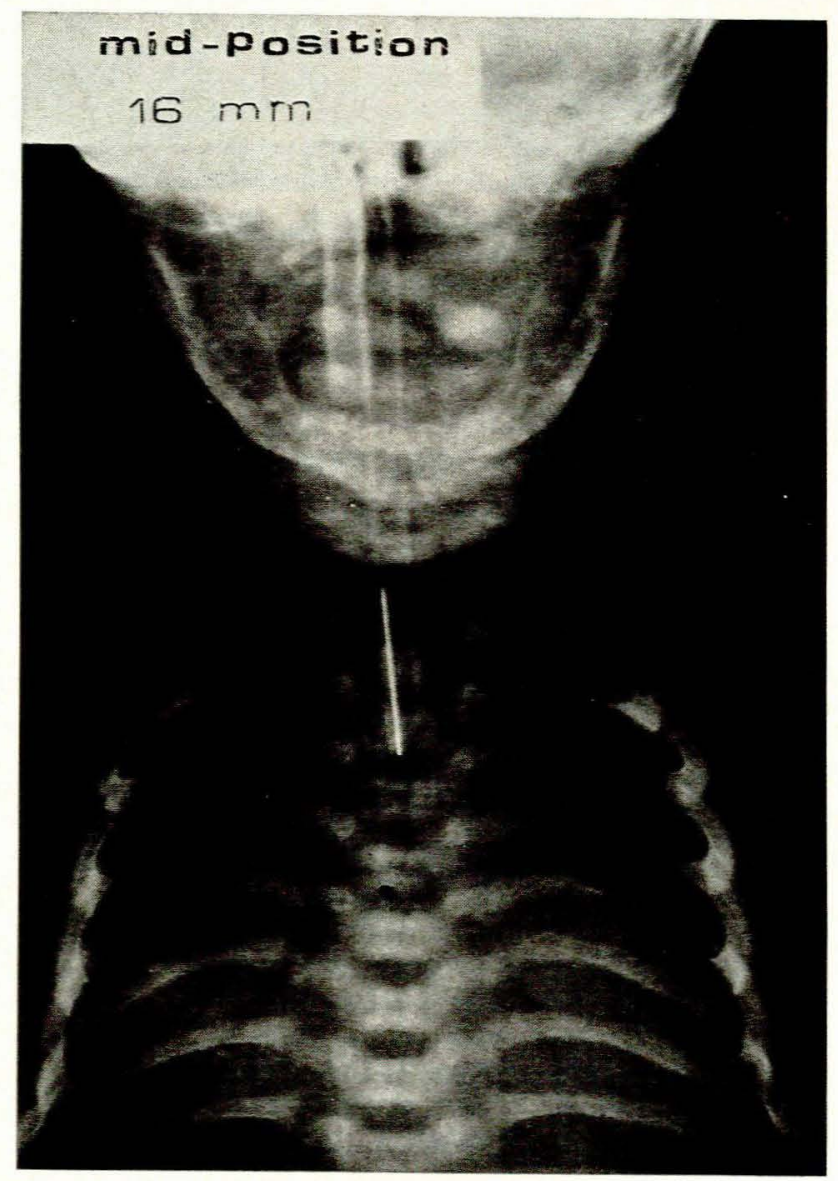

Fig. 1. Head in mid-position - the distance from the tube tip to the carina is $16 \mathrm{~mm}$. 


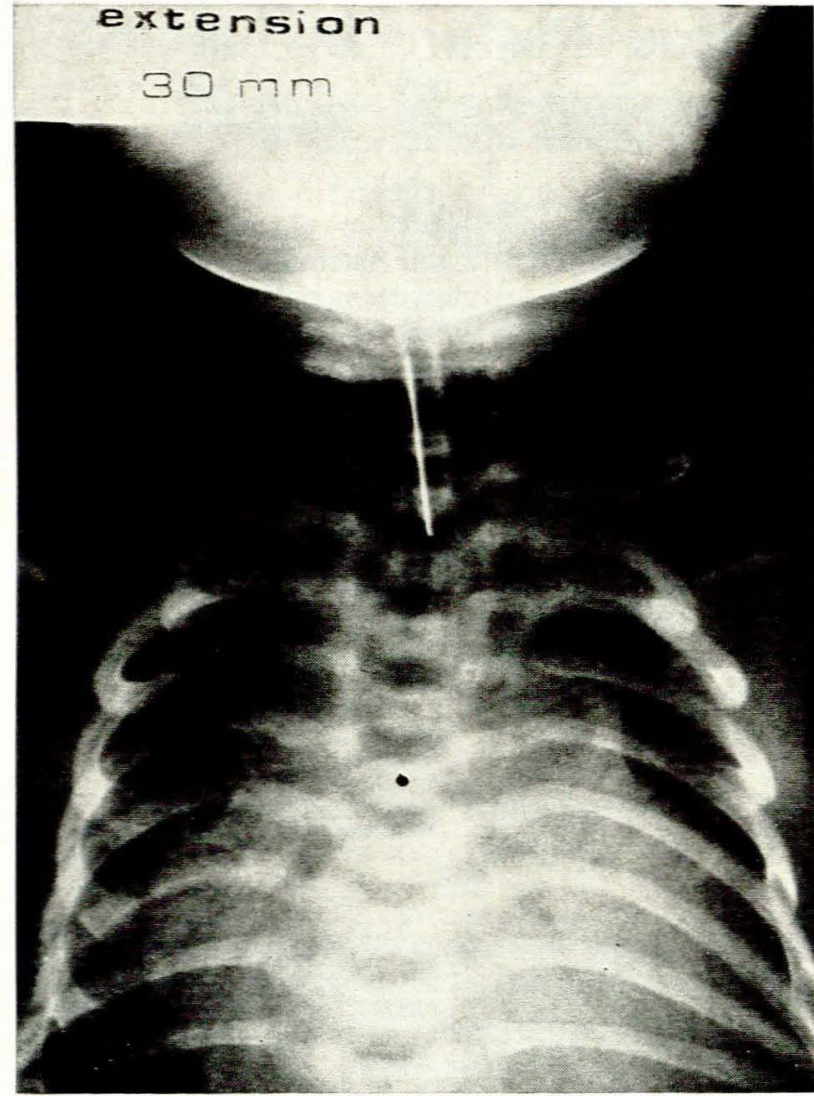

Fig. 2. Head in full extension - the distance from the tube tip to the carina is $30 \mathrm{~mm}$.

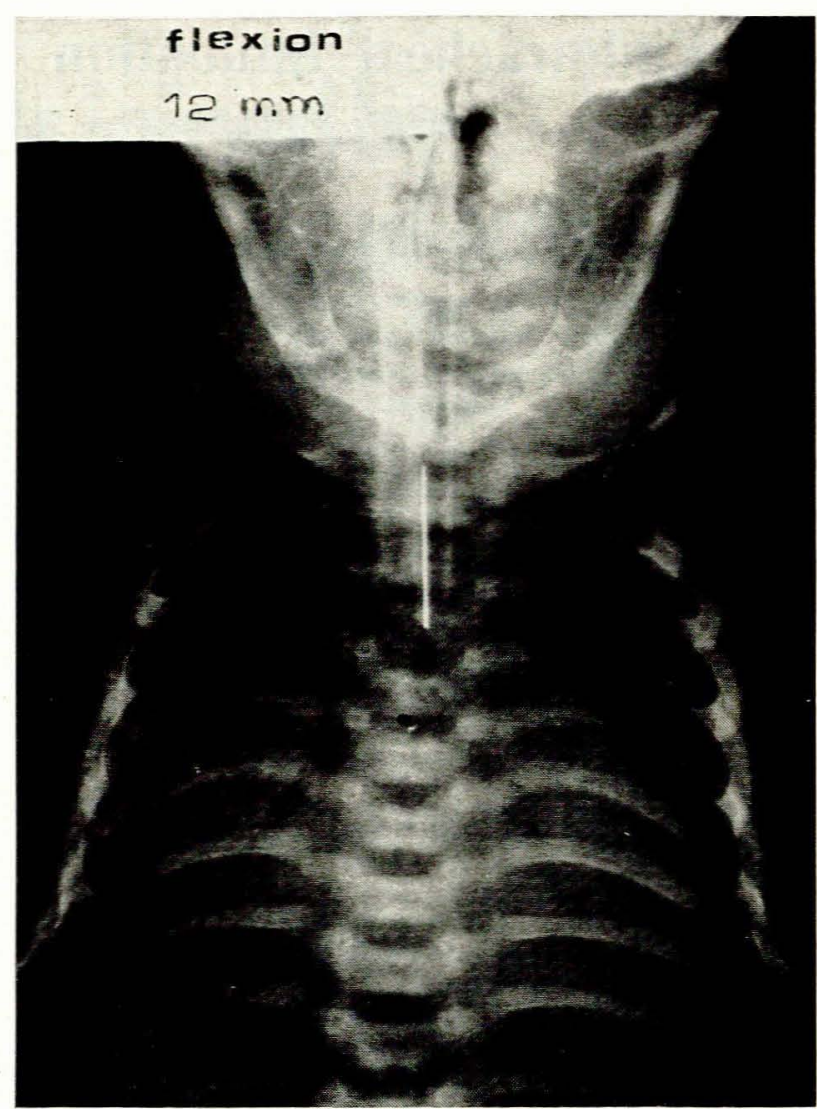

Fig. 3. Head in full flexion - the distance from the tube tip to the carina is $12 \mathrm{~mm}$.

TABLE I. DISTANCES $(\mathrm{mm})$ BETWEEN THE TIP OF THE ENDOTRACHEAL TUBE AND THE CARINA IN TWO INFANTS

\begin{tabular}{lrrcc} 
& \multicolumn{4}{c}{ Nasal intubation } \\
\cline { 2 - 5 } & Flex. & Ext. & Rot. & Mid-pos. \\
Infant 1 & 12 & 30 & 20 & 16 \\
Infant 2 & 5 & 23 & 14 & 15
\end{tabular}

\begin{tabular}{cccc}
\multicolumn{4}{c}{ Oral intubation } \\
\hline Flex. & Ext. & Rot. & Mid-pos. \\
4 & 12 & 6 & 7 \\
4 & 12 & 8 & 6
\end{tabular}

\section{RESULTS AND DISCUSSION}

The distances between the tip of the endotracheal tube and the carina with the infants in the various positions are shown in Table I.

The radiographs show that movement of the head can be a contributory cause of both malposition and displacement of the endotracheal tube in infants, either by accidental extubation or endobronchial intubation. If the infant is intubated with the head in extension as is usual, movement of the tube towards the carina must be anticipated when the head is moved to the mid-position. The position of the head must be known when correct placement of the tube is assessed radiologically.

In adults, Conrardy et $a l^{5}$. found that the tube moved an average of $19 \mathrm{~mm}$ towards the carina during flexion from a neutral position, and $19 \mathrm{~mm}$ away from the carina during extension, i.e. a total movement of $38 \mathrm{~mm}$. During rotation of the head, the average movement was $7 \mathrm{~mm}$ away from the carina. There was no difference between oral and nasal intubation. The maximum movement of $80 \mathrm{~mm}$ was seen between full flexion and extension.

\section{Safe Techniques}

With endotracheal intubation one must be certain that the tube is not placed too far into the trachea and that it is not in the oesophagus. On the other hand, it must obviously be far enough into trachea. Failure to ensure correct placement of the tube is a common error in intubation.

Oesophageal intubation is usually obvious when, on inflation, the stomach is being ventilated and not the lungs. In any case, routine auscultation of both lung fields in the lateral axillary line is mandatory.

Scanlon ${ }^{6}$ recommends two further 'quick' tests in neonates in specially equipped intensive care units. The first test requires that a naso-oesophageal tube be passed 


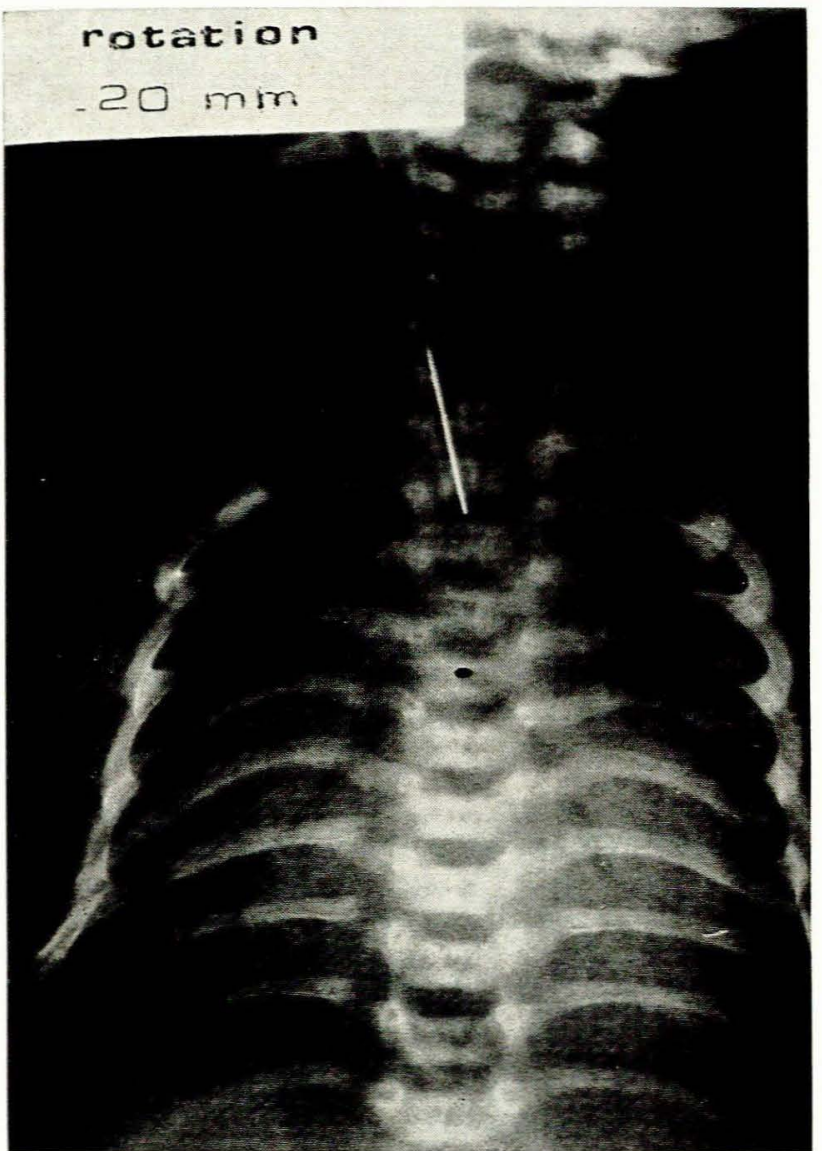

Fig. 4. Head in rotation - the distance from the tube tip to the carina is $20 \mathrm{~mm}$.

while $100 \%$ oxygen is given via the endotracheal tube, a sample of air is withdrawn from the oesophageal tube and tested with the Beckman model D2 Oxygen Analyser. If more than $50 \%$ oxygen is present, then oesophageal intubation has occurred. Resuscitation with $100 \%$ oxygen and a mask can give a transient false-positive result. If the patient has a nasotracheal tube in place, then the oesophagus is intubated orally.

In the second test $0,5 \mathrm{ml}$ methylene blue solution is injected into an oesophageal feeding tube, followed immediately by gentle and rapid syringe aspiration of the endotracheal tube. If any methylene blue is aspirated, the tube is in the oesophagus. Subsequent checks for malposition are done radiologically.

Several precautions can be taken to ensure that the endotracheal tube is put in far enough but not too far. The use of stepped endotracheal tubes (Coles pattern) is thought by some to prevent endobronchial intubation. It is too thick to be used nasally and many doctors do not use this kind of tube and doubt its efficacy. Auscultation of the chest is commonly done, and has been shown to be unreliable in diagnosing endobronchial intubation in infants ${ }^{2}$ (and adults).

Coldiron, ${ }^{7}$ who intubated dead infants, showed a good correlation between the distance of the nares to the "midtracheal point' and weight, head circumference and crown- heel length (crown-heel length in $\mathrm{cm} \times 0,21=$ tube length in $\mathrm{cm}$ ). Some workers use one and a half times the distance between the angle of the mouth and the tragus as the formula. Measurements and formulae are impractical, especially when emergency resuscitation is done by inexperienced operators and approximations are used. Kuhns and Poznanski ${ }^{3}$ recommended that a $9-\mathrm{cm}$ distance should be marked on, say, the operator's index finger. For 1-yearold infants $2 \mathrm{~cm}$ should be added and for very small premature infants $2 \mathrm{~cm}$ should be subtracted.

Bednarek and Kuhns ${ }^{4}$ describe a method of suprasternal palpation to ensure that the tube is in the endotracheal position and not inserted too far. The method proved reliable in their hands, while they had a persistently high rate of endobronchial intubation when they used other precautions, such as chest auscultation and measurement of tube length before intubation. The technique demands the use of a stilette to make palpation possible. The authors were able, with this method, to place the tube close to the 'interclavicular mid-point', which they showed corresponded very closely with the mid-point of the trachea. The best precaution is to use a neonatal tube which has a circumferential mark $\pm 2,2 \mathrm{~cm}$ from its tip. Loew and Thibeault ${ }^{5}$ recommended marks 2,2, 2,4 and $2,6 \mathrm{~cm}$ from the tip in tubes with diameters of $2,5,3,0$ and $3,5 \mathrm{~mm}$, respectively. Others recommend this procedure, but such tubes are not readily available. The average length of the trachea in the living neonate is $5,7 \mathrm{~cm}$ according to one author. ${ }^{3}$ In dead infants (38. 43 weeks), where some tissue shrinkage is said to occur, the length is given as between 4,0 and $4,77 \mathrm{~cm}^{7,9}$ Intubation is done under vision. It should be possible to introduce the marker on the tube as far as the vocal cords in full-term neonates, to above the vocal cords in premature infants, and to slightly below in older infants.

The long-term prevention of malposition depends on regular radiography.

\section{CONCLUSIONS}

Because the distance between the glottis and carina is so small in infants, care and accuracy in intubation are essential. In the neonate this distance is not likely to be less than $40 \mathrm{~mm}$ and movement not greater than $18 \mathrm{~mm}$. Thus it is advisable to mark each tube $22 \mathrm{~mm}$ from its tip, and to fix the tube when this mark has reached the vocal cords. Fixation of the tube to the lower lip may be safer than fixation to the upper lip. In this way one avoids endobronchial intubation and total dislodgement. Particular care should be taken with procedures such as pneumoencephalography, oesophagoscopy and head and neck surgery, and with conditions such as posterior fossa tumours of the brain.

\section{REFERENCES}

\footnotetext{
1. Hamilton, N. and Stevens, N. (1966): J. Amer. med. Ass., 198, 1113. Heinonen, J., Takki, S. and Tammisto, T. (1969): Lancet, 1, 850. Kuhns, L. R. and Poznanski, A. K. (1971): J. Pediat., 78, 991. Bednarek, F. J, and Kuhns, L. R. (1975): Pediatrics, 56, 224. Conrardy, P. A., Goodman, L. R., Lainge, F. et al. (1976): Crit. Care Med., 1, 8. 